\title{
Optimization of Transparent Conducting Oxides for Liquid Crystal Based Optical Phased Arrays
}

\author{
John D. Perkins, Charles W. Teplin, Maikel. F.A.M. van Hest, \\ Chollada Warmsingh and David S. Ginley \\ National Renewable Energy Laboratory \\ 1617 Cole Blvd. \\ Golden, CO 80401 \\ 303-384-6606 \\ john_perkins@nrel.gov \\ Scott R. Harris \\ ARFL/SNJM \\ 3109 P Street Bldg 622 \\ WPAFB, OH 45433-7700 \\ 937-255-9614 x285 \\ Scott.Harris@wpafb.af.mil
}

\begin{abstract}
Transparent conducting oxides are used as a contacting layer to liquid crystal (LC) based single wavelength optical phased array (OPA) infrared beam steerers. To investigate TCO optimization for this application, a numerical model based on the Drude (freeelectron) theory is used to calculate the optical absorption at $1550 \mathrm{~nm}$ for layers with a fixed sheet resistance, $\mathrm{R}_{\mathrm{S}}$, as a function of conducting electron concentration and carrier mobility. For fixed $R_{s}$, carrier mobility is found to be the critical parameter. In short, increased mobility allows for reduced carrier concentration and hence less absorption for a given sheet resistance. For $\mathrm{R}_{\mathrm{s}}=3000 \Omega / \mathrm{Sq}$., as is used in current prototype LC-OPA devices, the use of high conductivity commercial ITO would require using a very thin, $\sim 1 \mathrm{~nm}$ thick, ITO layer. However, the conventionally worse TCO $\mathrm{Zn}-\mathrm{Sn}-\mathrm{O}$ has good mobility but with a much lower carrier concentration than ITO which allows for a more robust layer thickness of $20 \mathrm{~nm}$ while still keeping the absorption minimal.
\end{abstract}

\section{INTRODUCTION}

Optical phased array (OPA) technology is being developed as a non-mechanical replacement for gimbaled mirrors in beam steering applications [1-3]. These infrared beam steering devices operate in an analogous fashion to microwave frequency phased array radar antennas. In one approach these optical phased arrays (OPAs) employ a nematic liquid crystal layer as an electrically addressable optical phase shifting array. Typically, a transparent conducting oxide (TCO) film is deposited on an optical window to provide electrical contacts to the liquid crystal layer. The TCOs must meet a complex set of requirements for transparency at the design wavelength, conductivity, and optical damage resistance. In particular, many proposed applications require the ability to handle high optical powers $\left(\sim 170 \mathrm{MW} / \mathrm{cm}^{2}\right)$ at $1550 \mathrm{~nm}$. Conventional materials such as commercial Indium-Tin-Oxide (ITO) and Al doped $\mathrm{ZnO}(\mathrm{ZnO}: \mathrm{Al})$ are not well optimized for these applications. These transparent conducting oxide (TCO) coatings are generally optimized for applications such as laptop computers or solar cells where high visible wavelength transparency and low sheet resistances are desired. For nematic liquid crystal OPA applications and the devices examined so far, sheet resistances of up to $3000 \Omega / \mathrm{Sq}$. are acceptable since this value results in an electrical RC time constant that is sufficiently shorter than the relaxation time of the liquid crystal layer being addressed. In addition, minimizing the absorption at the operating wavelength $(1550 \mathrm{~nm})$ is critical to avoid possible thermal damage to the contact layer. We discuss here a numerical model based on the Drude (free-electron) theory for the electrical conductivity and infrared optical properties of TCO materials for OPA applications. This model has been used to calculate the absorption at $1550 \mathrm{~nm}$ for layers with a fixed sheet resistance as a function of conducting electron concentration and carrier mobility. For the case of fixed sheet resistance at $3000 \Omega / \mathrm{Sq}$., we find that the use of high quality commercial ITO, which has been optimized for reduced absorption in the visible and small sheet resistances, would require prohibitively thin $(\sim 1 \mathrm{~nm})$ ITO layers. Because the absorption at $1550 \mathrm{~nm}$ is dominated by free carrier electrons, our model shows that the critical parameter for this application is the carrier mobility. In short, increased mobility allows for reduced carrier

${ }^{1}$ U.S. Government work not protected by U.S. copyright.

2 IEEEAC paper \#1540, Updated December 16, 2002 


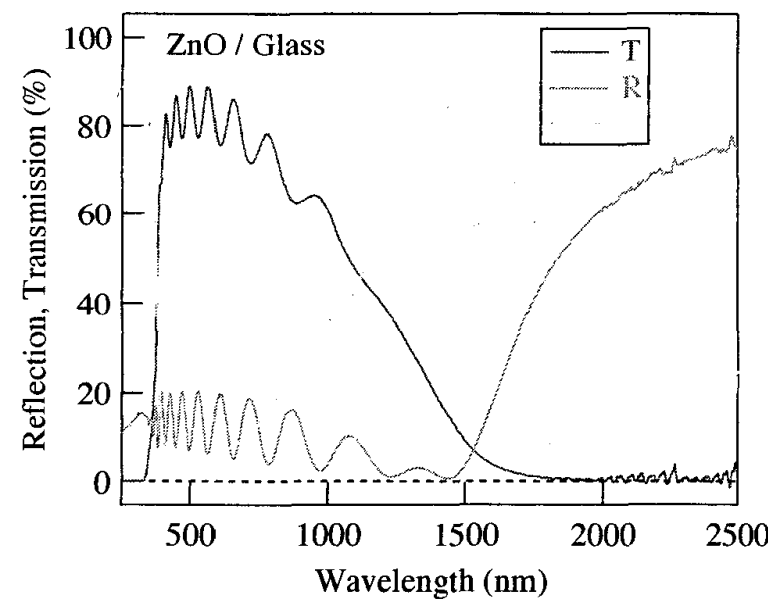

Figure 1 - Optical reflection (red), transmission (blue) and absorption (green) of a typical $\mathrm{ZnO}$ TCO film optimized for use in the visible.

concentration and hence less absorption for a given sheet resistance. Fortunately, we find that the TCO $\mathrm{Zn}-\mathrm{Sn}-\mathrm{O}$ which is too resistive for conventional applications fits these requirements and allows for a more robust layer thickness of $20 \mathrm{~nm}$.

\section{Optical Properties of typical TCO's}

Figure 1 shows optical transmission $(T)$, reflection $(R)$ and absorption (A) spectra for a typical $\mathrm{ZnO} \mathrm{TCO}$. In the visible region, $\sim 500 \mathrm{~nm}$, the film is quite transparent, $80 \%$, with pronounced wavelength oscillations in the transmission due to interference among multiple internal reflections within the weakly absorbing TCO layer. At shorter wavelengths, $\sim 350 \mathrm{~nm}$, the abrupt drop in the transmission is due to the main band gap transition. At longer wavelengths, $\sim 1500 \mathrm{~nm}$, the more gradual decrease in the transmission and increase in the reflection with increasing wavelength is due to absorption by and reflection from the conduction band electron plasma oscillations. For this sample, the peak absorption occurs at $\sim 1500 \mathrm{~nm}$, the plasma wavelength as indicated in the figure. Also, the maximum absorption is quite strong, $\sim 80 \%$. In short, this TCO is quite transparent in the visible but not in the spectral range of interest for OPA applications. This example, then, clearly shows need to address TCO optimization in an application specific way.

The plasma frequency, which in TCOs marks the IR cross over from transparent to reflecting, increases as the square root of the carrier concentration. In particular,

$$
\omega_{p}=\sqrt{\frac{4 \pi N e^{2}}{\varepsilon_{\infty} m^{*}}}
$$

where $\mathrm{N}$ is the conducting electron concentration, e the electron charge, $\varepsilon_{\infty}$, the high frequency (visible wavelength) dielectric constant and $\mathrm{m}^{*}$, the electron effective mass. In

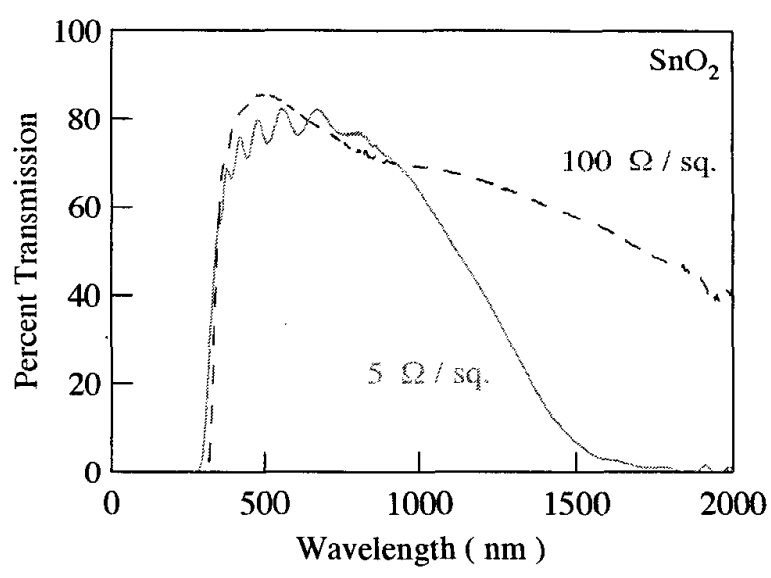

Figure 2 - Effect of conductivity on IR transmission of a TCO.

terms of wavelength, the plasma wavelength, $\lambda_{p}$, goes roughly as

$$
\lambda_{p} \sim \sqrt{\frac{m^{*}}{N}} .
$$

So as the carrier concentration, and hence typically the conductivity, are increased, the plasma edge moves to shorter wavelengths. This can be seen in Figure 2 which shows the transmission spectra for two $\mathrm{SnO}_{2}$ films with two different sheet resistances, $\mathrm{R}_{\mathrm{s}}$. For the more conducting sample (lower $R_{S}$ ) with $R_{S}=5 \Omega / S q$, the long wavelength cut off in the transmission occurs at shorter wavelengths than for the less conducting sample with $\mathrm{R}_{\mathrm{S}}=100 \Omega / \mathrm{Sq}$.

Figure 3 shows the reflection and transmission the spectra for the $\mathrm{ZnO}$ on glass $\mathrm{TCO}$ substrate used in the current prototype LC OPA devices [3]. The transmission spectrum is measured from 250 to $6000 \mathrm{~nm}$. In the transmission spectrum, the strong absorption feature at $\sim 2700 \mathrm{~nm}$ and

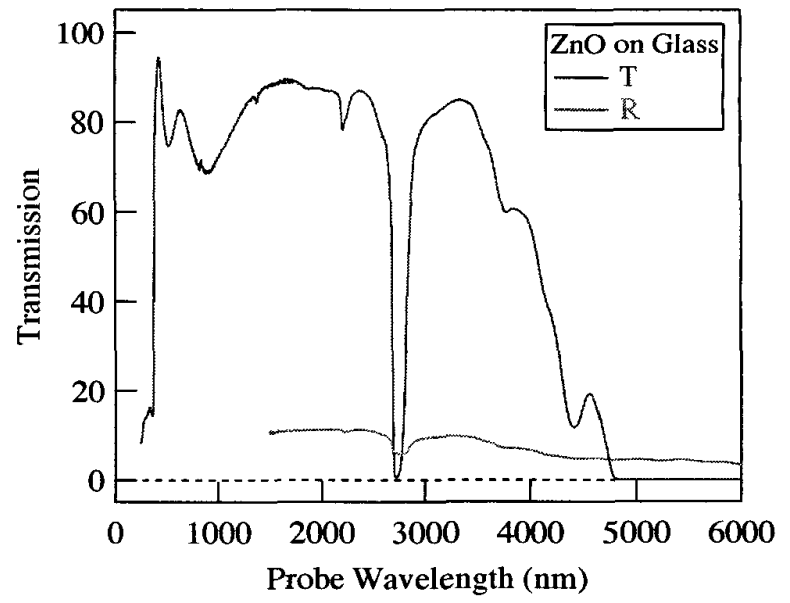

Figure 3 - Reflection (red) and transmission (blue) of $\mathrm{ZnO}$ test film used in prototype OPAs. 


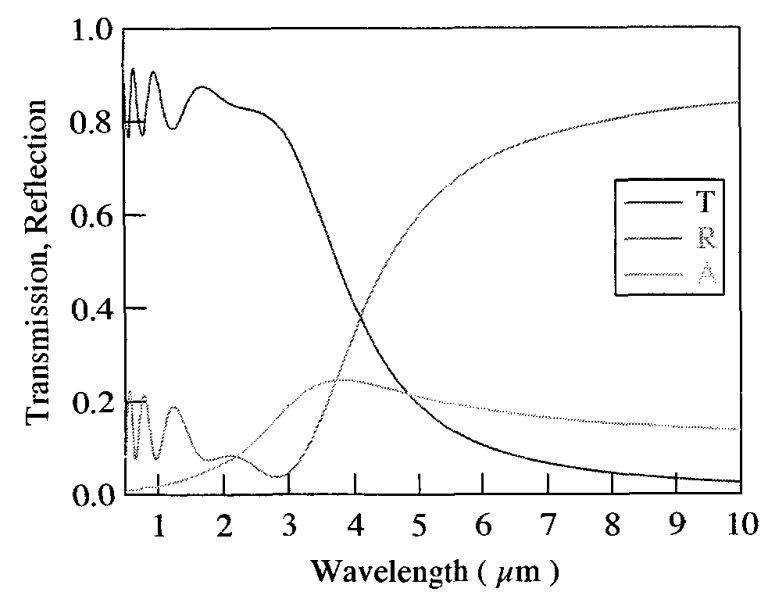

Figure 4 - Calculated reflection (red), transmission (blue) and absorption (green). Model parameters: $\mathrm{N}=$ $1 \times 10^{20} \mathrm{~cm}^{-3}, \mu=100 \mathrm{~cm}^{2} / \mathrm{V}-\mathrm{s}, \mathrm{m}^{*}=0.3 \mathrm{~m}_{e}, \mathrm{~d}=500$ $\mathrm{nm}, \varepsilon_{\infty}=4$.

the nearly monotonic drop off in transmission between 3000 and $5000 \mathrm{~nm}$ are both just features of the glass substrate. The very abrupt decrease in transmission at $\sim 300 \mathrm{~nm}$ is the band gap absorption and a few interference oscillations can be seen in the visible and near-IR region. The reflection spectrum is nearly constant at $\sim 10 \%$ over the measured region from 1500 to $6000 \mathrm{~nm}$. Taken together, these reflection and transmission spectra resemble those for a nonconducting dielectric layer on glass rather than those of a highly doped TCO as in Figures 1 and 2.

For the $0.3 \mu \mathrm{m}$ thick sample in Figure 3, the measured sheet resistance is $3000 \Omega /$ sq. giving a resistivity, $\rho$, of $\rho=$ $9 \times 10^{-2} \Omega-\mathrm{cm}$. Room temperature Hall measurements give a carrier concentration, $\mathrm{N}$, of $\mathrm{N}=5 \times 10^{18} \mathrm{~cm}^{-3}$ and a mobility, $\mu$, of $\mu=13.6 \mathrm{~cm}^{2} / \mathrm{V}$-s. For highly conducting Al doped $\mathrm{ZnO}, \rho=1.3 \times 10^{-4} \Omega$-cm and $\mathrm{N}=1.5 \times 10^{2}$ $\mathrm{cm}^{-3}$ [4]. Hence, for the $\mathrm{ZnO}$ films used in the prototype MC OPAs, the carrier concentration and conductivity are already quite low in comparison with conventional TCOs.

In prototype OPA beam steerers, these $\mathrm{ZnO}$ layers have shown visible damage upon routine use at $1560 \mathrm{~nm}$ with $0.17 \mathrm{~J} / \mathrm{cm}^{2}, 3 \mathrm{~ns}$ pulses at $10 \mathrm{~Hz}$. The current design goal is for sustained operation at $0.5 \mathrm{~J} / \mathrm{cm}^{2}$ per pulse. At present it is not known whether the damage in the current devices is due to the intrinsic TCO absorption, point defects in the TCO or device dependent interfacial issues. Nevertheless, minimizing the intrinsic TCO absorption at the operating wavelength should be beneficial. Hence, we have developed and used a simple model describing the electrical and IR optical properties of TCO materials to evaluate if a different choice of TCO material could result in less absorption at $1550 \mathrm{~nm}$ and therefore hopefully less damage.

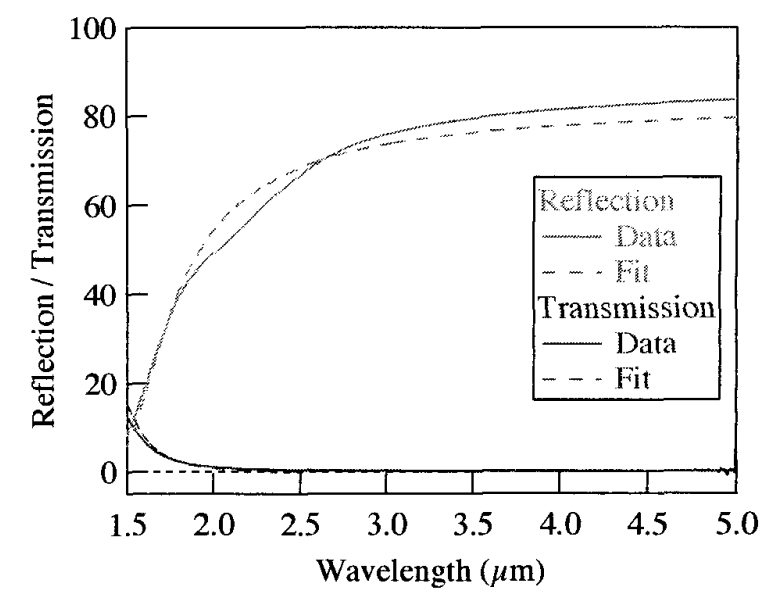

Figure 5 - Fit of model to Cd-Sn-O TCO film. Fit parameters: $\omega_{\mathrm{p}}=6272 \mathrm{~cm}^{-1}, \tau=4.1 \times 10^{-15} \mathrm{~s}, \varepsilon_{\infty}=4, \mathrm{~d}$ $=603 \mathrm{~nm}$. Reflection spectra in red, transmission spectra in blue. Dashed lines are fits to solid line data.

\section{Modeling Optical Properties of TCO's}

The infrared optical properties of TCOs can be well modeled using a simple Drude free-electron model [5]. In this model the frequency dependent complex dielectric constant, $\varepsilon(\omega)$, is given by

$$
\varepsilon(\omega)=\left[1-\left(\frac{\omega_{p}}{\omega}\right)^{2} \frac{1}{1+(i / \omega \tau)}\right]
$$

where $\tau$ is the electron scattering time. The optical constants $\mathrm{n}$ and $\mathrm{k}$ are easily calculated from Eq. 3 as

$$
\begin{aligned}
& n=\operatorname{Re}(\sqrt{\varepsilon(\omega)}) \\
& \kappa=\operatorname{Im}(\sqrt{\varepsilon(\omega)}) .
\end{aligned}
$$

Using these optical constants one can calculate the reflection and transmission spectra for relatively simple geometries assuming purely specular reflection with no scattering due to imperfect interfaces. In this approximation the absorption, A, is just 1-R-T.

Figure 4 shows the calculated reflection, transmission and absorption spectra for light normally incident upon a simple TCO layer on a lossless dielectric slab substrate with $\mathrm{n}=$ 1.5 (the index of refraction for glass in the visible and near IR). The model includes coherent reflections within the film as well as incoherent reflection from the backside substrate-air interface. The model does not include a band gap absorption and hence is only applicable to examining the IR optical properties of TCO materials. For the spectra shown in this figure, the model parameters are $\mathrm{N}=1 \times 10^{20}$ $\mathrm{cm}^{-3}$, mobility $\mu=100 \mathrm{~cm}^{2} / \mathrm{V}-\mathrm{s}, \mathrm{m}^{*}=0.3 \mathrm{~m}_{\mathrm{e}}$, thickness $\mathrm{d}$ $=500 \mathrm{~nm}$ and $\varepsilon_{\infty}=4$. The mobility can be determined from the effective mass and the scattering time by 


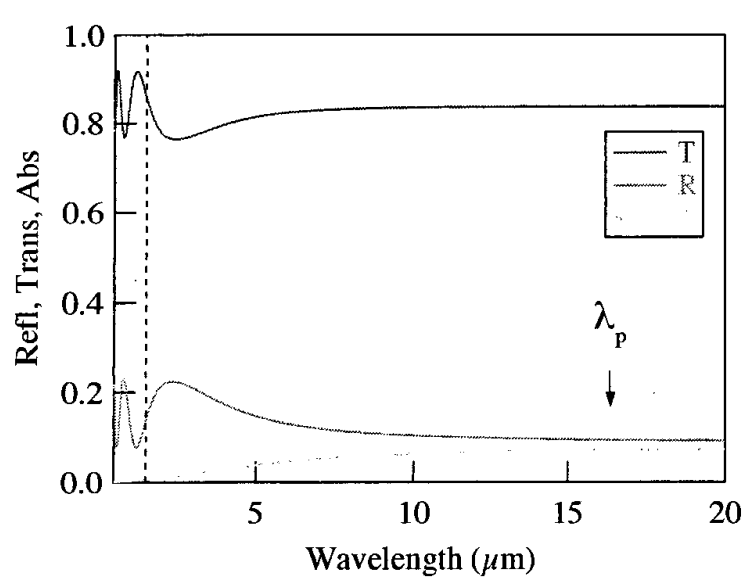

Figure 6 - Calculated spectra using measured electrical parameters for $\mathrm{ZnO}$ test film. Model parameters: $\mathrm{N}=$ $5 \times 10^{18} \mathrm{~cm}^{-3}, \mu=13.6 \mathrm{~cm}^{2} / \mathrm{V}-\mathrm{s}, \mathrm{m}^{*}=0.3 \mathrm{~m}_{\mathrm{e}}, \mathrm{d}=300$ $\mathrm{nm}, \varepsilon_{\infty}=4$. Reflection spectra in red, transmission in blue, absorption in green.

$$
\mu=\frac{e \tau}{m^{*}} .
$$

Therefore, using equations 1 and 5 , one can determine $\left(\omega_{\mathrm{p}}\right.$, $\tau)$ from $\left(\mathrm{N}, \mu, \mathrm{m}^{*}, \varepsilon_{\infty}\right)$ and hence evaluate the dielectric function using equation 3. Excepting the band gap absorption, the modeled spectra shown in Figure 4 show all the expected TCO optical features for a conventional TCO such as that shown in Figure 1. These are interference oscillations in the visible and near-IR where the TCO layer is transparent as well as the coupled decrease in transmission and increase in reflection with increasing wavelength due to plasma oscillations and the associated absorption peak. It is these latter plasma oscillation related properties which are the focus of the Drude model used here. Figure 5 shows a fit (dashed line) using this model to the measured (solid line) IR reflection and transmission for a
Cd-Sn-O TCO film on glass [6].

As previously discussed, over the measured spectral range, the reflection and transmission spectra for our $\mathrm{ZnO}$ test film shown in Figure 3 do not show the correlated decrease in transmission and increase in reflection characteristic of the plasma edge region of a TCO film. Nevertheless, one can use the experimentally measured carrier concentration, $\mathrm{N}=5$ x $10^{18} \mathrm{~cm}^{-3}$, mobility, $\mu=13.6 \mathrm{~cm}^{2} / \mathrm{V}$-s and thickness, $\mathrm{d}=$ $300 \mathrm{~nm}$, to calculate the TCO related optical properties. The resultant calculated reflection, transmission and absorption spectra are shown in Figure 6 with the additional necessary parameters set at $\mathrm{m}^{*}=0.3 \mathrm{~m}_{\mathrm{e}}$ and $\varepsilon_{x}=4$ [5]. Note that the spectra range for Figure 6 extends to $20 \mu \mathrm{m}$. For these spectra; the plasma wavelength, indicated in the figure with a vertical arrow, is $16.3 \mu \mathrm{m}$, much longer than the OPA operating wavelength of $1.55 \mu \mathrm{m}$ which is indicated by the vertical dashed black line. Hence, the calculated absorption at $1.55 \mu \mathrm{m}$ is quite weak, just $0.6 \%$, and the sample is quite transparent at $1.5 \mu \mathrm{m}$ in good general agreement with the measured spectra shown in Figure 3.

The plasma oscillation related absorption is peaked at the plasma wavelength and the basic width of the absorption on the short wavelength side depends strongly upon the mobility [5]. Thus increasing the mobility makes the plasma oscillation absorption narrower on the short wavelength side which reduces the absorption at wavelengths substantially shorter than the plasma wavelength. Hence, the results of Figure 6 suggest that if the mobility could be increased, there would be less absorption at $1.5 \mu \mathrm{m}$.

To further explore the effect of mobility and carrier concentration on the TCO optical properties at $1550 \mathrm{~nm}$, we have used the model to make $2 \mathrm{D}$ color intensity maps, which show the reflection, transmission and absorption at $1550 \mathrm{~nm}$ as a function of the carrier concentration and mobility. Figure 7 shows one such set of maps. Note that the vertical axis is the log of the carrier concentration and
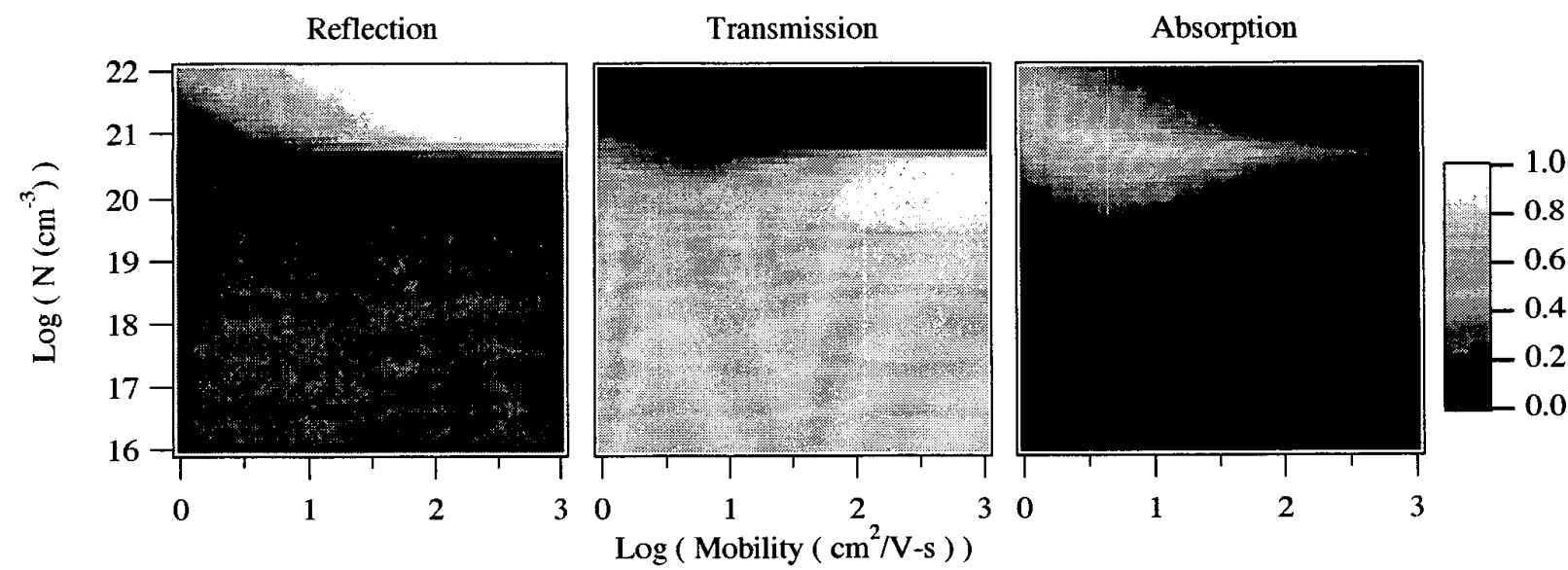

Figure 7 - Reflection, Transmission and Absorption at $1550 \mathrm{~nm}$ for fixed film thickness $\mathrm{d}=500 \mathrm{~nm}$. Other model parameters: $\mathrm{m}^{*}=0.3 \mathrm{~m}_{\mathrm{e}}, \varepsilon_{\infty}=4$. 

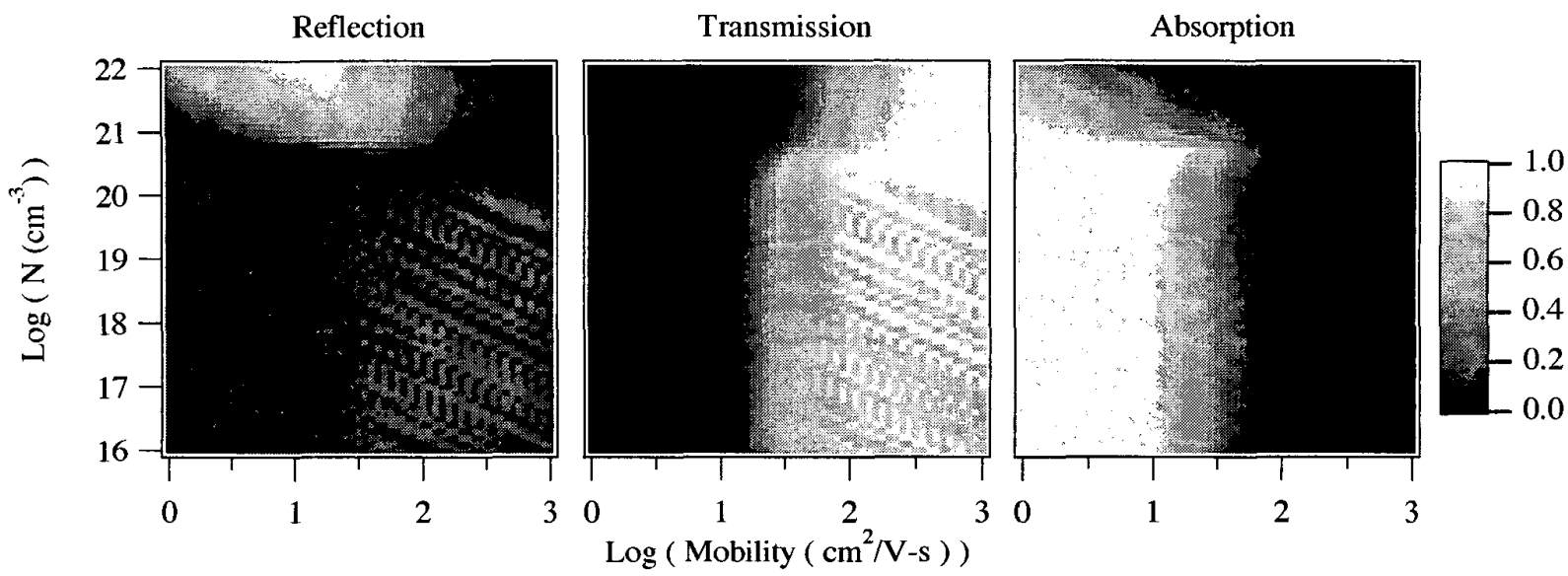

Figure 8 - Reflection, Transmission and Absorption at $1550 \mathrm{~nm}$ for fixed sheet resistance $\mathrm{R}_{\mathrm{S}}=10 \Omega / \mathrm{Sq}$. Other model parameters: $\mathrm{m}^{*}=0.3 \mathrm{~m}_{\mathrm{e}}, \varepsilon_{\infty}=4$.

the horizontal axis is the $\log$ of the mobility. Hence these maps cover 6 orders of magnitude in $\mathrm{N}$ and 3 orders of magnitude in $\mu$. A yellow-hot color scale is used and unless otherwise noted, the color scale range is 0 to 1 . For all the maps shown in Figures $7,8,9$ and $10, \mathrm{~m}^{*}=0.3 \mathrm{~m}_{\mathrm{e}}$ and $\varepsilon_{\infty}=4$.

The $1550 \mathrm{~nm} \mathrm{R,T}$, and A maps shown if Figure 7 are calculated for a fixed thickness film with $\mathrm{d}=500 \mathrm{~nm}$. The most pronounced feature of this map set is the abrupt increase in reflection and decrease in transmission with increasing carrier concentration at $\log (N) \approx 21$. This is easily understood. From Equation 2 one sees that as the carrier concentration increases the plasma wavelength decreases. In particular, with $\mathrm{m}^{*}=0.3 \mathrm{~m}_{\mathrm{e}}$ and $\varepsilon_{\infty}=4$, the carrier concentration which gives a plasma wavelength of $1550 \mathrm{~nm}$ is $\mathrm{N}_{1550}=5.6 \times 10^{20} \mathrm{~cm}^{-3}$ or $\log \left(\mathrm{N}_{1550}\right)=20.7$. Hence, as the carrier concentration increases past $\mathrm{N}_{1550}$, the plasma wavelength moves from being longer than $1550 \mathrm{~nm}$ to shorter than $1550 \mathrm{~nm}$ and hence at $1550 \mathrm{~nm}$ the sample goes from being mostly transparent to mostly reflecting. The absorption map shows that there is, in general, only strong absorption at $1550 \mathrm{~nm}$ when the carrier concentration is such that the plasma wavelength is not too far from 1550 $\mathrm{nm}$ as expected from the measured and modeled spectra shown in Figures 1 and 4 respectively.

However, while the color maps in Figure 7 are straightforward to understand by comparison with modeled and measured spectra, they are not useful in developing improved TCOs for OPA applications. Fixed thickness is not a good constraint for evaluating TCOs for use in OPAs, LEDs or a solar cells. Since the electrical conductivity, o, depends on the product of the carrier concentration and the mobility as

$$
\sigma=N e \mu
$$

there are large changes in the conductivity at different $(N, \mu)$ pairs and hence, as in Figure 7, for a fixed thickness there are big changes in the sheet resistance which is what determines the TCO related electrical resistance between the contact pads and the LC pixel being biased. In fact, from the upper right to the lower left corners of the Figure 7 maps, the sheet resistance increases by nine orders of magnitude.

Constant sheet resistance is a more appropriate constraint and Figure 8 shows $\mathrm{R}, \mathrm{T}$ and $\mathrm{A}$ maps calculated with the constraint $\mathrm{R}_{S}=10 \Omega / \mathrm{Sq}$. With this constraint, for each $(\mathrm{N}$, $\mu$ ) pair, the thickness is determined from

$$
d=\frac{1}{R_{S}} \cdot \frac{1}{N e \mu} .
$$

A comparison of the transmission maps shown in Figures 7 and 8 clearly shows the big difference between the constraint of constant thickness and the constraint of constant sheet resistance. In the former case, the boundary between transparent and opaque is nearly a horizontal line, with the carrier concentration being the most important parameter. In contrast, for the case of constant sheet resistance shown in Figure 8 , the boundary between transparent and opaque is roughly a broad vertically oriented line with mobility now being the most important parameter. This approximately $90^{\circ}$ reorientation of the boundary between transparent and opaque occurs because under the constraint of constant sheet resistance used in Figure 8, there are very large changes in the thickness of the TCO film which in turn has large effects on the net transmission, reflection and absorption.

The constant sheet resistance absorption map shown in Figure 8 shows that the key to making low absorption TCO films under this constraint is to increase the mobility. Figure 9 shows the constant sheet resistance $\mathrm{R}, \mathrm{T}$ and $\mathrm{A}$ maps for $\mathrm{R}_{\mathrm{S}}=3000 \Omega / \mathrm{Sq}$., similar to that for the $\mathrm{ZnO}$ test sample above. For this larger sheet resistance, the maps in Figure 9 show, to a first approximation, that for all $(N, \mu)$ pairs the $3000 \Omega / \mathrm{Sq}$. TCO films are not very reflective, are quite transparent and are not too absorbing. However, from Figure 6 , we found for the $\mathrm{ZnO}$ test film with $(\mathrm{N}, \mu)=$ 


\section{Vol. 4-1828}

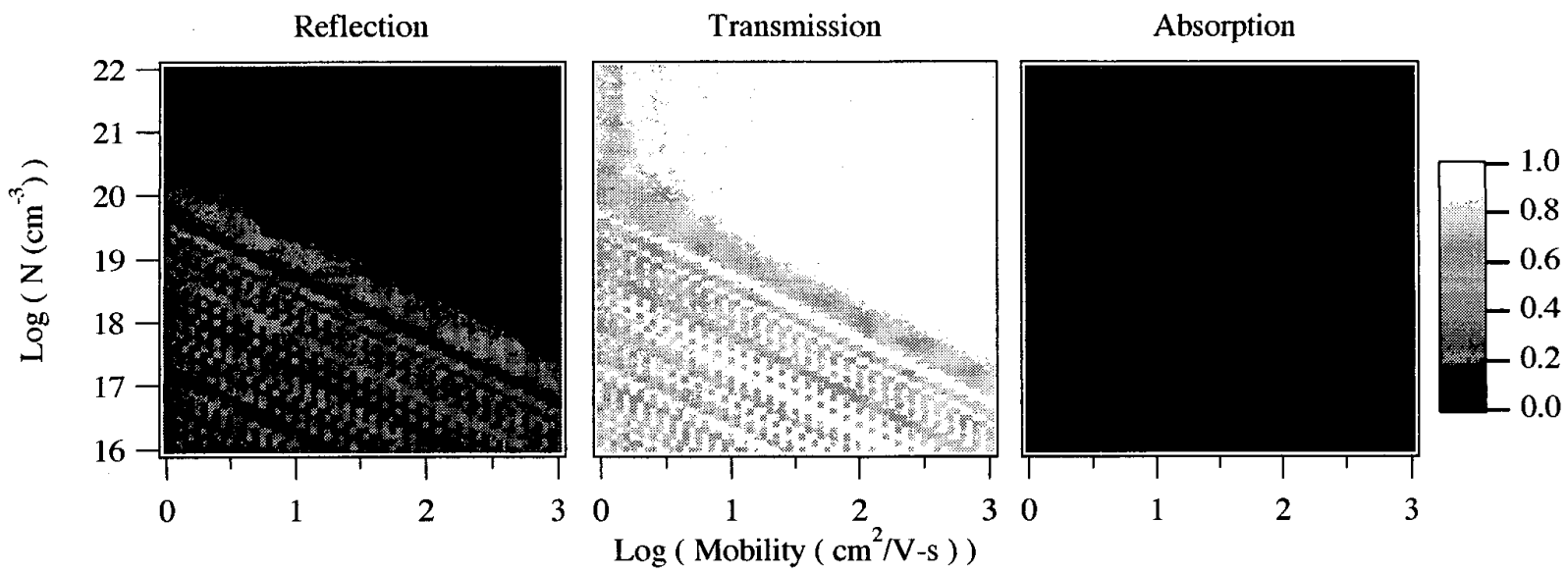

Figure 9 - Reflection, Transmission and Absorption at $1550 \mathrm{~nm}$ for fixed sheet resistance $\mathrm{R}_{\mathrm{S}}=300010 \Omega / \mathrm{Sq}$. Other model parameters: $\mathrm{m}^{*}=0.3 \mathrm{~m}_{\mathrm{e}}, \varepsilon_{\infty}=4$.

$\left(5 \times 10^{18}, 13.6\right)$ that the absorption was already small, only 0.006 in the units of Figure 9. Hence, while the full scale range of 0 to 1 in Figure 9 is useful for comparison with Figure 8 it is not useful for determining if TCOs exist with lower absorption at $1550 \mathrm{~nm}$ than the $\mathrm{ZnO}$ which is already being used.

Figure 10 shows the same absorption map for $\mathrm{R}_{\mathrm{S}}=3000$ $\Omega / \mathrm{Sq}$. from Figure 9 but now with the color scale covering only 0 to 0.02 ( $2 \%$ absorption), the region of interest for OPA applications. The green diagonal lines are contours of constant thickness labeled at the edges with the TCO film thickness in $\mathrm{nm}$. All absorption values higher than 0.02 are shown as the same solid yellow as the color scale maximum so most of the solid yellow region on the left side of Figure 10 is just off scale. With this choice of color scale range, one can see that like in Figure 8 , mobility is the most important parameter and that there is a meaningful decrease

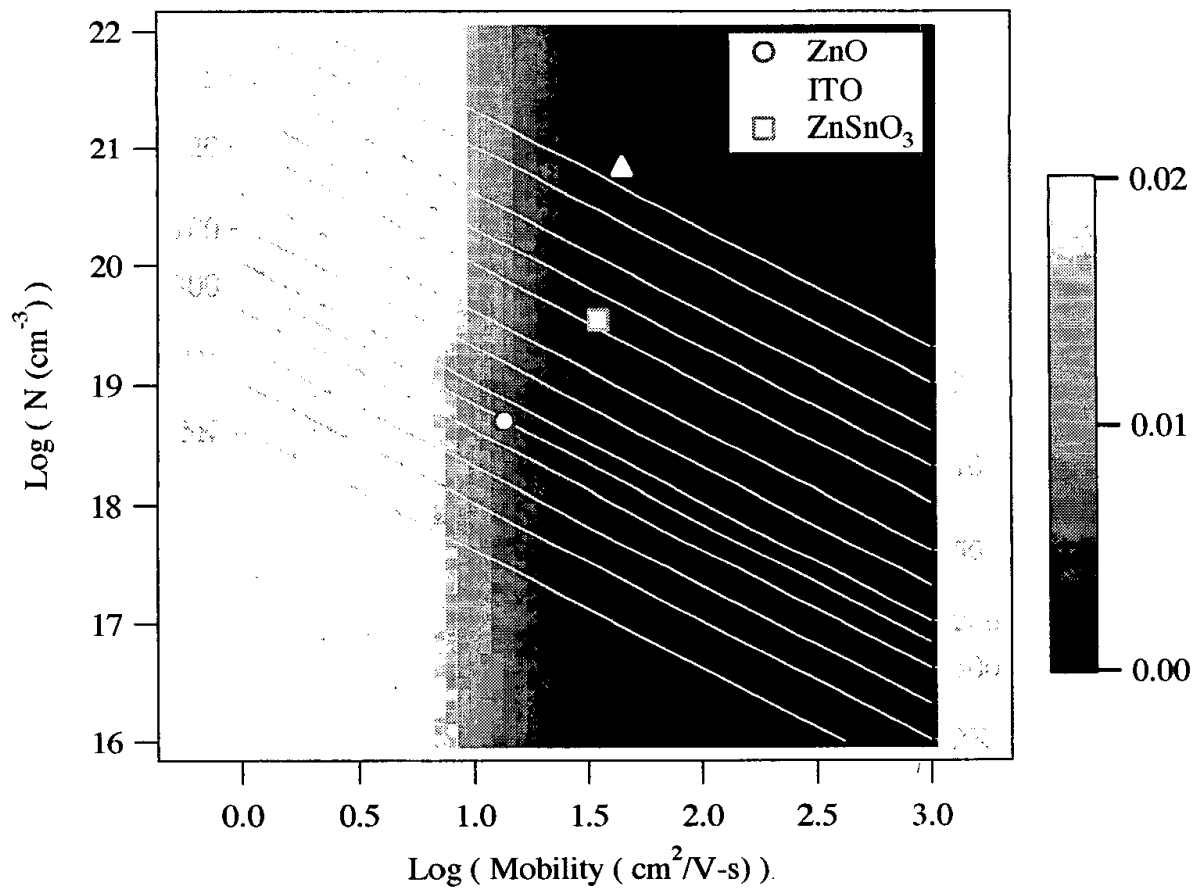

Figure 10 - Absorption at $1550 \mathrm{~nm}$ for fixed sheet resistance $\mathrm{R}_{\mathrm{S}}=300010 \Omega / \mathrm{Sq}$. Other model parameters: $\mathrm{m}^{*}=0.3$ $\mathrm{m}_{\mathrm{e}}, \varepsilon_{\infty}=4$. Diagonal green lines are contours of constant film thickness in nm. Note full scale is 0.02 ( $2 \%$ absorption). Off scale data is also plotted in same color as full scale value. 
in the absorption as the mobility is increased from $\sim 10$ ( $\log (\mu)=1)$ to $\sim 30 \mathrm{~cm}^{2} / \mathrm{V}-\mathrm{s}(\log (\mu)=1.5)$. This suggests that it should be possible to reduce the absorption at $1550 \mathrm{~nm}$ while keeping the electrical performance of the OPA the same.

To make this clear, the $(\mathrm{N}, \mu)$ pair for the $\mathrm{ZnO}$ test sample is plotted as a purple circle. The blue triangle shows the $(\mathrm{N}, \mu)$ pair for good commercially available ITO. While the high mobility of the ITO means that a less absorbing TCO layer could, in principle, be made using this very conducting material, the films would have to be very thin, less than $1 \mathrm{~nm}$. This is because of the high carrier concentration, $\mathrm{N} \approx 7 \times 10^{20} \mathrm{~cm}^{-3}$. If such ITO films were used, but were in actuality thicker, their sheet resistance would be substantially lower than $3000 \Omega / \mathrm{Sq}$. and hence the results of Figure 10 would simply not apply. Note that since the absorption is exponential in the film thickness, the difference between $1 \mathrm{~nm}$ and $2 \mathrm{~nm}$ would quite significant if $1 \mathrm{~nm}$ is the necessary thickness to meet the constant sheet resistance constraint. The red square shows the $(N, \mu)$ pair for an NREL grown $\mathrm{ZnSnO}_{3}$ sample which also has a higher mobility than the $\mathrm{ZnO}$ test sample, but for which $\mathrm{N}$ is 10 times smaller than the ITO samples. Hence, the necessary thickness to meet the constant sheet resistance constraint is $20 \mathrm{~nm}$, a much more practical thickness for a TCO thin film. According to this model, such a $\mathrm{ZnSnO}_{3}$ would have the same sheet resistance as the $\mathrm{ZnO}$ test film but with less absorption at $1550 \mathrm{~nm}$.

\section{Conclusion}

We have developed a numerical model based on the Drude (free-electron) theory for the electrical conductivity and infrared optical properties of TCO materials. This model has been used to calculate the absorption at $1550 \mathrm{~nm}$ for layers with a fixed sheet resistance as a function of conducting electron concentration and carrier mobility. For the case of fixed sheet resistance at $3000 \Omega / \mathrm{Sq}$., we find that the use of high quality commercial ITO, which has been optimized for reduced absorption in the visible and small sheet resistances, would require a very thin, $\sim 1 \mathrm{~nm}$ thick, ITO layer for optimum performance in OPA applications. Because the absorption at $1550 \mathrm{~nm}$ is dominated by free carrier electrons, our model shows that the critical parameter for this application is the carrier mobility. In short, increased mobility allows for reduced carrier concentration and hence less absorption for a given sheet resistance thus improving OPA performance. NREL developed TCO films in the $\mathrm{Zn}-\mathrm{Sn}-\mathrm{O}$ system appear to have the desired combination of conductivity and transparency at $1550 \mathrm{~nm}$ with a more robust layer thickness of $20 \mathrm{~nm}$. The model developed appears to be an important tool for the development of optimum OPA structures.

\section{REFERENCES}

[1] P. McManamon, E. Watson, T. Dorschner and L. Barnes, "Applications look at the use of liquid crystal writeable gratings for steering passive radiation," Optical Engineering 32, 2657, 1993.

[2] P.F. McManamon et al., "Optical phased array technology," Proceedings of the IEEE 84, 268, 1996.

[3] S.R. Harris, "Characterization and application of a liquid crystal beam steering device," Proceedings of the SPIE - The International Society for Optical Engineering 4291, 109, 2001.

[4] T. Minami, "New n-Type Transparent Conducting Oxides," Mater. Res. Soc. Bull. 25, 38, 2000.

[5] T.J. Coutts, D.L. Young and X. Li, "Characterization of Transparent Conducting Oxides," Mater. Res. Soc. Bull. 25, 58, 2000.

[6] X. Li et al., "A Comparison of Composite Transparent Conducting Oxides Based on the Binary Compounds $\mathrm{CdO}$ and $\mathrm{SnO}_{2}, " \mathrm{NCPV}$ Program Review Proceedings: $N C P V$ Program Review, 2001.

John Perkins is a Senior Scientist at the National Renewable Energy Laboratory where he is a member of the Electronic Materials and Devices Division of the National Center for Photovoltaics. His current research focuses on the material science and deposition of mixed metal oxides and nitrides for energy related technologies. He received a Ph.D. in Physics from M.I.T. in 1994. 